\title{
Thermal properties of black and blue phosphorenes from a first-principles quasiharmonic approach
}

\author{
Yierpan Aierken, ${ }^{1, *}$ Deniz Çakır, ${ }^{1, \dagger}$ Cem Sevik, ${ }^{2, \dagger}$ and Francois M. Peeters ${ }^{1, \S}$ \\ ${ }^{1}$ Department of Physics, University of Antwerp, Groenenborgerlaan 171, B-2020 Antwerpen, Belgium \\ ${ }^{2}$ Department of Mechanical Engineering, Faculty of Engineering, Anadolu University, Eskisehir, TR 26555, Turkey
}

(Received 6 July 2015; published 19 August 2015)

\begin{abstract}
Different allotropes of phosphorene are possible of which black and blue phosphorus are the most stable. While blue phosphorus has isotropic properties, black phosphorus is strongly anisotropic in its electronic and optical properties due to its anisotropic crystal structure. In this work, we systematically investigated the lattice thermal properties of black and blue phosphorene by using first-principles calculations based on the quasiharmonic approximation approach. Similar to the optoelectronic and electronic properties, we predict that black phosphorene has highly anisotropic thermal properties, in contrast to the blue phase. The linear thermal expansion coefficients along the zigzag and armchair direction differ up to $20 \%$ in black phosphorene. The armchair direction of black phosphorene is more expandable as compared to the zigzag direction and the biaxial expansion of blue phosphorene under finite temperature. Our comparative analysis reveals that the inclusion of finite-temperature effects makes the blue phase thermodynamically more stable over the black phase above $135 \mathrm{~K}$.
\end{abstract}

DOI: 10.1103/PhysRevB.92.081408

A monolayer of phosphorus, known as phosphorene, has been successfully exfoliated [1,2] and was introduced as a new member of the two-dimensional (2D) crystal family. Triggered by this realization, various physical properties of phosphorene were explored. Structurally, at least six different possible stable two-dimensional crystal structures were proposed for phosphorene [3-5]. Among those, black phosphorene (black P) is the most stable allotrope. However, the cohesive energy of blue phosphorene (blue $\mathrm{P}$ ) is only a few $\mathrm{meV}$ higher than that of black $\mathrm{P}$, while other crystal structures are much less favorable with energy at least by $\sim 80 \mathrm{meV} /$ atom higher. Electronically, black $\mathrm{P}$ is a semiconductor with a direct band gap at the $\Gamma$ point $[3,6]$. Experimentally, photoluminescence excitation spectroscopy found a quasiparticle band gap of $2.2 \mathrm{eV}$ [7], which is large when compared to its bulk band gap $0.31-0.33 \mathrm{eV}[8,9]$. Due to its electronic structure, black P has been proposed as a potential novel material in nanoelectronics and optoelectronics, especially in the infrared regime. Highperformance black $\mathrm{P}$ based transistors with a mobility up to $1000 \mathrm{~cm}^{2} / \mathrm{V} \mathrm{s}$ and an on/off ratio up to $10^{4}$ at room temperature have been reported recently $[1,2]$.

Similar to black $\mathrm{P}$, blue $\mathrm{P}$ was predicted to be a semiconductor material with an indirect band gap around $2.00 \mathrm{eV}$ [3]. Therefore, it can be of potential use for field-effect transistor (FET) applications. These studies points towards a promising future for these phosphorene materials. However, a more comprehensive knowledge of finite-temperature effects on their properties is urgently needed and could contribute to an acceleration in the progress towards these application. While the electronic [3,4,10-14], vibrational [15-17], and optical [6,18-23] properties together with anisotropic thermal conductivity $[24,25]$ of mono- and multilayer phosphorene

\footnotetext{
*yierpan.aierken@uantwerpen.be

†dcakir79@gmail.com

${ }_{\ddagger}^{\ddagger}$ csevik@anadolu.edu.tr

§francois.peeters@uantwerpen.be
}

PACS number(s): 65.80.-g, 65.40.De, 63.22.Np

have been investigated previously, the thermal expansion, temperature dependence of free energy, and specific heat of black and blue $\mathrm{P}$ are yet unknown. This motivated us to execute a first-principles study of the thermal properties, i.e., temperature-dependent lattice constant, thermal expansion coefficients, free energy, and specific heat, for both black $\mathrm{P}$ and blue P.

Our first-principles calculations are performed within the density functional theory (DFT) as implemented in the Vienna ab initio Simulation Package (VASP) [26-29]. A projected augmented wave (PAW) method $[30,31]$ and a plane wave basis set with a cutoff energy of $500 \mathrm{eV}$ are used. The exchange-correlation interaction is described with the generalized gradient approximation (GGA) within the Perdew-BurkeErnzerhof (PBE) formulation [32,33]. Brillouin zones of black $\mathrm{P}$ and blue $\mathrm{P}$ are sampled in the Monkhorst-Pack scheme with $15 \times 11 \times 1$ and $15 \times 15 \times 1 k$-point grids, respectively. These values are symmetrically reduced to the corresponding $k$-point grids for a supercell by keeping the $k$-point density the same. A supercell with a $25 \AA$ interlayer distance is introduced to prevent interlayer interactions. Force criteria for ionic step is set to $10^{-7} \mathrm{eV} / \AA$ for the phonon calculations. Force constant matrices used in the phonon calculations are calculated by density functional perturbation theory using the VASP code, in which we use a supercell with dimension of $7 \times 3 \times 1$ and $5 \times 5 \times 1$ for black $\mathrm{P}$ and blue $\mathrm{P}$, respectively. Phonon frequencies and corresponding thermodynamic properties (on a $200 \times 200 \times 1$ converged $q$-point mesh) are obtained by the PHONOPY [34] program.

Equilibrium lattice constants at any temperature $\alpha(T)$ are calculated by direct minimization of the Helmholtz free energy $F(\alpha, T)$ with respect to its independent lattice vector $\alpha$, i.e., $a$ and $b$ for black P, and $a$ for blue $\mathrm{P}$ (using the quasiharmonic approximation [35-37] based PHONOPY-QHA script [38]). For the minimization process, $F(\alpha, T)$ is obtained by fitting the discrete data points of $F\left(\alpha_{i}, T\right)$ to the third-order BirchMurnaghan equation of state, where $i$ is the label for different lattice constants or equivalently different strains. The $F\left(\alpha_{i}, T\right)$ is constructed from $\omega_{\boldsymbol{q}, j}^{i}$ and $E\left[\alpha_{i}\right]$ through the following 

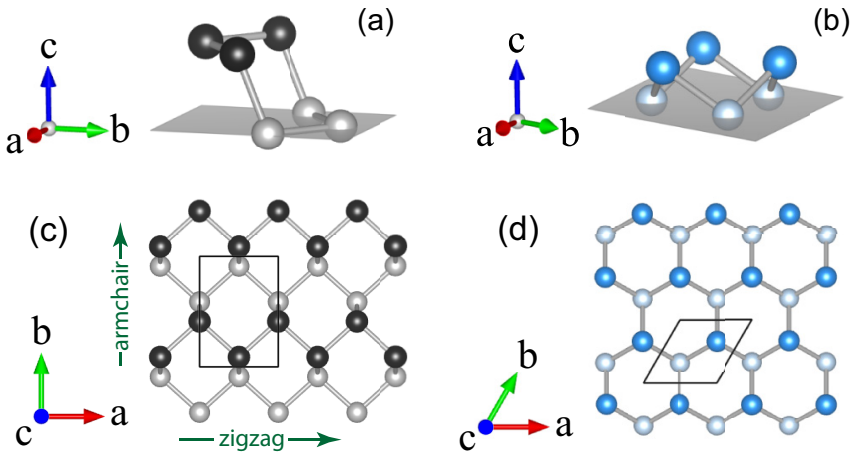

FIG. 1. (Color online) (a) and (b) are the one hexagonal ring of black $\mathrm{P}$ and blue $\mathrm{P}$; their top viewed structures are shown in (c) and (d), respectively. Atoms are colored according to structure's name; lighter colored atoms mean lower in vertical position. Black boxes in top views are the primitive unit cell used in our calculations.

formula $[35,39]$ :

$$
\begin{aligned}
F\left(\alpha_{i}, T\right)= & E\left[\alpha_{i}\right]+\sum_{\boldsymbol{q}, j} \frac{\hbar \omega_{\boldsymbol{q}, j}^{i}}{2} \\
& +k_{B} T \sum_{\boldsymbol{q}, j} \ln \left(1-\exp \left[-\frac{\hbar \omega_{\boldsymbol{q}, j}^{i}}{k_{B} T}\right]\right) .
\end{aligned}
$$

Here, $T$ is the temperature, $k_{B}$ is the Boltzmann constant, and $E\left[\alpha_{i}\right]$ is the DFT ground state energy. $\omega_{\boldsymbol{q}, j}^{i}$ is the phonon frequency at the $q$ point $q$ with band index $j$. The sums run over all wave vectors and all bands of the whole Brillouin zone. Since structural instabilities arise especially for the armchair direction when under compressive strain values larger than $4 \%$, the calculation of phonon dispersions for both structures is performed under small strains, namely $\pm 2 \%$, in order to evaluate $\omega_{\boldsymbol{q}, j}^{i}$ and $E\left[\alpha_{i}\right]$. Considering two independent lattice vectors $\mathbf{a}$ and $\mathbf{b}$ of black $\mathrm{P}$, two uniaxial strains are applied for these directions, while for blue $\mathrm{P}$, only biaxial strain is applied to keep its hexagonal symmetry unchanged.

The thermal expansion coefficient $\alpha(T)$ is then calculated through the following formula:

$$
\alpha(T)=\frac{1}{a(T)} \frac{d a(T)}{d T},
$$

where $a(T)$ is the equilibrium lattice parameter corresponding to the minimum of the Helmholtz free energy.

Different from pure planar graphene, black $\mathrm{P}$ and blue $\mathrm{P}$ have a buckled nonplanar structure due to $s p^{3}$ hybridization, yet all three structures share the same hexagonal lattice base. Given an almost identical unit cell structure, see Fig. 1, it is not surprising that blue $\mathrm{P}$ and black $\mathrm{P}$ have similar total energy. The only significant difference is the plane marked as gray shown in Fig. 1, on which phosphorus atoms extend to form an infinite $2 \mathrm{D}$ crystal. Therefore, thermal expansion on these different planes is expected to be different and will carry insight information on the different finite-temperature properties. The primitive unit cell of black $P$ is a rectangular lattice with a four-atom basis and a space group of $D_{2 h}^{7}$, and that of blue $\mathrm{P}$ is a hexagonal lattice with a two-atom basis and a space group of $D_{3 d}^{3}$. Therefore, except for the three acoustic modes from the in-phase vibrations of atoms in the unit cell for both structures, out-of-phase vibrations of the atoms result in nine and three optical modes for black $\mathrm{P}$ and blue $\mathrm{P}$, respectively.

The calculated phonon dispersions corresponding to these modes are depicted in Figs. 2(a) and 2(b), along with the high symmetric $q$ points of both structures. Parallel with the previous calculations $[15,40]$, the calculated phonon dispersions are free from imaginary frequencies, which ensures the structural stability of the materials. The lowest acoustic mode ZA (out-of-plane transversal vibration) displays a $q^{2}$ relation due to the bending of the monolayer instead of the shearing found in the bulk counterpart [41]. Such flexural modes are expected to be unstable at finite temperatures and are responsible, e.g., for the intrinsic ripples in graphene [42] and $\mathrm{MoS}_{2}$ [43]. The other two acoustic modes (in-plane transversal and longitudinal vibration) still have a linear dependence with respect to the $q$ wave vector since the situation is the same here as in bulk. The total frequency range of the phonon dispersion
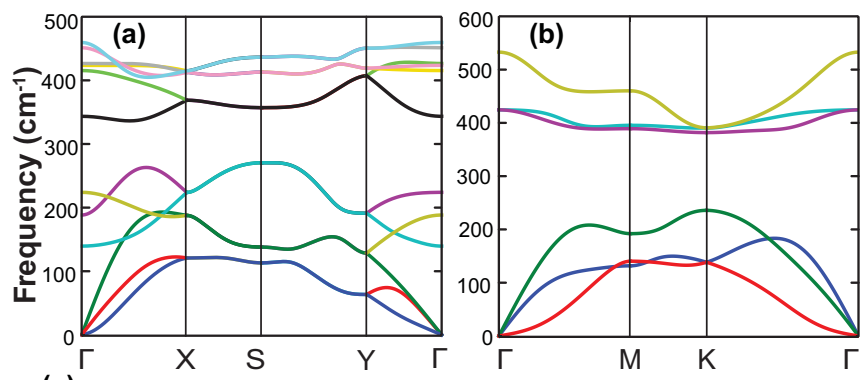

(c)
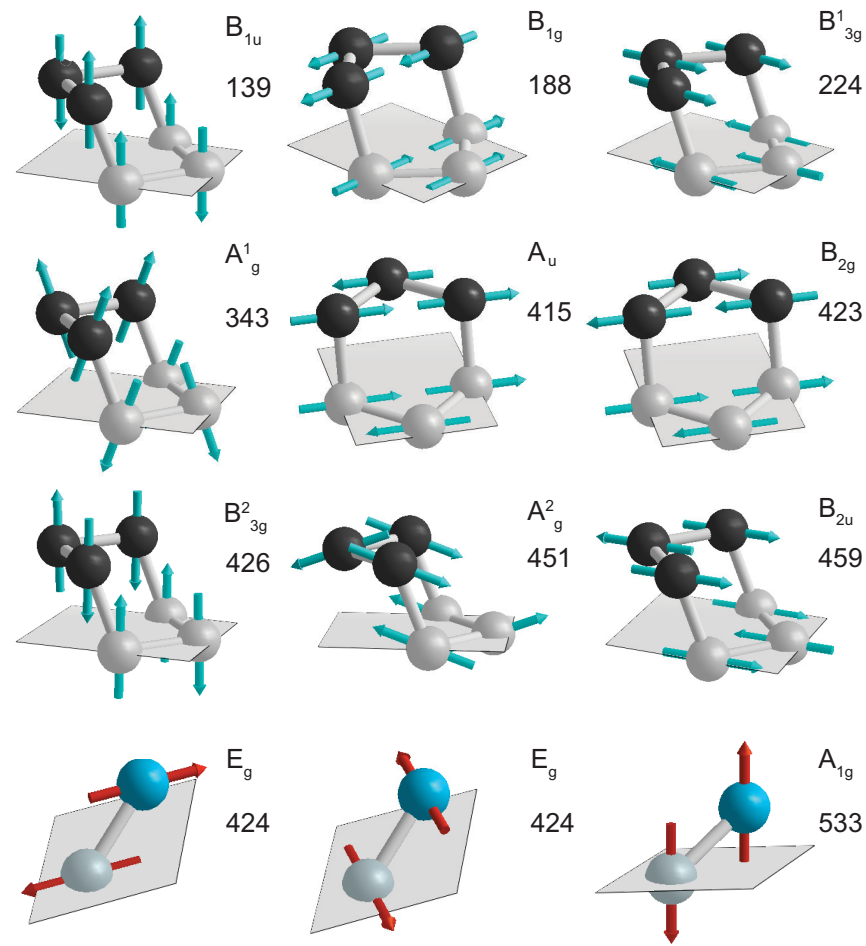

FIG. 2. (Color online) Calculated phonon dispersions for strainless (a) black and (b) blue P. (c) Optical phonon modes together with their frequencies (in units of $\mathrm{cm}^{-1}$ ) at the $\Gamma$ point and irreducible representations for black $\mathrm{P}$ and blue $\mathrm{P}$. 

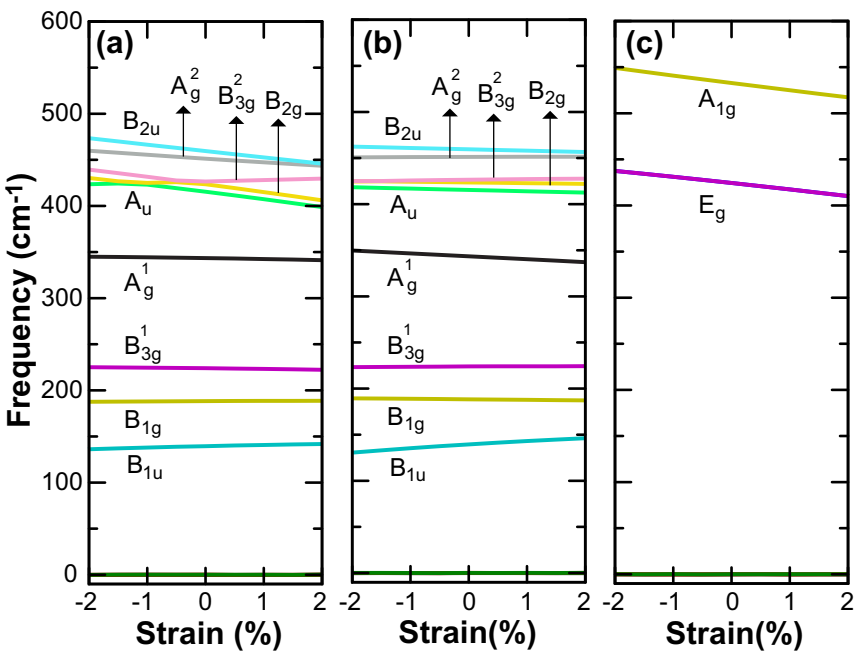

FIG. 3. (Color online) Variation of the $\Gamma$ frequencies with strain: (a) zigzag and (b) armchair direction of black $\mathrm{P}$ and (c) blue $\mathrm{P}$.

is larger by an amount of about $100 \mathrm{~cm}^{-1}$ in blue $\mathrm{P}$ as compared to black P.

With the knowledge of the space group and its character table it is possible to assign vibrational modes to each band at the $\Gamma$ point. A table of all optical modes together with its frequency and irreducible representation for both structures is presented in Fig. 2(c) for the strainless structures. In Fig. 3, we show the variation of these optical phonons at the $\Gamma$ point under uniaxial strain. The frequency shift by strain can be directly detected using Raman spectroscopy. Depending on the atomic motion of the modes and the magnitude and type of strain, we find that the dependence of the phonon frequency can be different for each mode. Especially, the higher energy optical modes under uniaxial strain, applied along the zigzag direction, exhibit a much larger variation; see Fig. 3(a). The $B_{3 g}^{2}$ mode of black $\mathrm{P}$ has a different behavior under tensile and compressive strains along the zigzag direction. Applying a tensile (compressive) strain increases (decreases) the frequency of the $B_{3 g}^{2}$ mode. The frequency spacing between the $A_{g}^{2}$ and $B_{2 u}$ modes decreases when black $\mathrm{P}$ is stretched along both zigzag and armchair directions. While the frequency of the $A_{g}^{2}$ mode exhibits almost no variation under strain applied along the armchair direction, it significantly varies under strain applied along the zigzag direction. Among the low-lying optical modes (i.e., $B_{1 u}, B_{1 g}$, and $B_{3 g}^{1}$ ), the $B_{1 u}$ mode exhibits a larger variation with applied strain. The $E_{g}$ and $A_{1 g}$ modes of blue $\mathrm{P}$ always are redshifted when changing strain from compressive to tensile. Our results show that by measuring the frequency shift in the particular modes, it is possible to determine the strain distribution in phosphorene.

The equilibrium lattice constants, $a_{0}$ and $b_{0}$ of black $\mathrm{P}$ and $a_{0}$ of blue $\mathrm{P}$, are predicted as $a_{0}=3.298 \AA, b_{0}=4.625 \AA$, and $a_{0}=3.277 \AA$, respectively, in good agreement with previously reported results $\left(a_{0}=3.297 \AA, b_{0}=4.640 \AA\right.$ for black $\mathrm{P}$ $[6,13]$, and $a_{0}=3.330 \AA$ for blue P [3]). The expansion of these lattice parameters due to zero-point vibration is around $0.2 \%$ at $0 \mathrm{~K}$, which is smaller than that of other well-known two-dimensional materials such as graphene and $h-\mathrm{BN}$, but

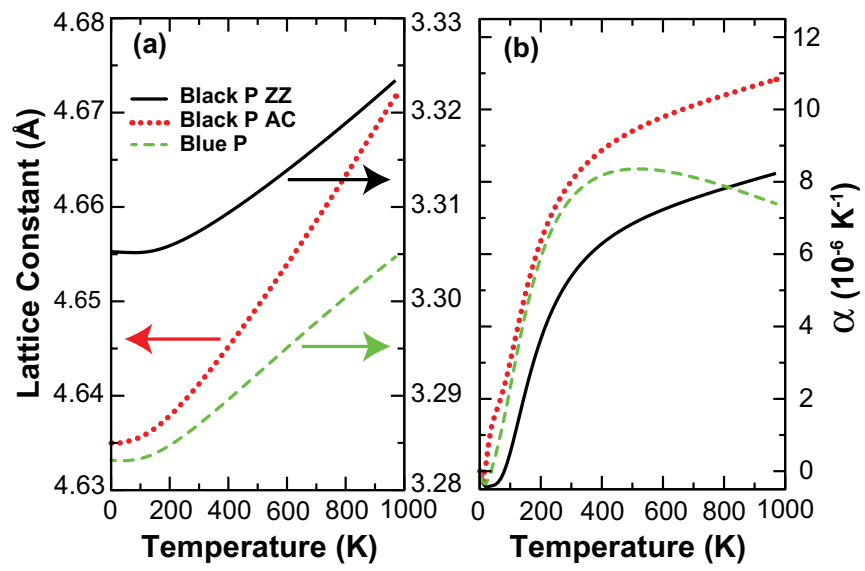

FIG. 4. (Color online) (a) Lattice constants and (b) thermal expansion coefficients as a function of temperature. Here, $\mathrm{ZZ}$ and $\mathrm{AC}$ are used for the zigzag and armchair directions, respectively.

it is comparable with that of $\mathrm{MoS}_{2}$ and $\mathrm{MoSe}_{2}$. This result is reasonable due to the difference in the maximum phonon frequency between these materials: that of graphene and $h$-BN is around $1600 \mathrm{~cm}^{-1}$ and that of $\mathrm{MoS}_{2}, \mathrm{MoSe}_{2}$, black P, and $a_{0}$ of blue $\mathrm{P}$ is around $600 \mathrm{~cm}^{-1}$.

The temperature dependence of the lattice constants $a(T)$, $b(T)$, and thermal expansion coefficients (TEC) $\alpha(T)$ of both structures are shown in Fig. 4. The anisotropic nature of the structure of black P leads to different lattice constant expansion rates. A faster thermal expansion along the armchair direction (i.e., b) is found; see Fig. 4(a). While a small negative TEC appears for all structures in all directions at temperatures lower than $100 \mathrm{~K}$, black $\mathrm{P}$ along the zigzag direction has a more apparent negative expansion. The lattice constant of both phases varies linearly when $T>200 \mathrm{~K}$; see Fig. 4(a). The TEC increases rapidly with temperature up to $300 \mathrm{~K}$. After that, all TEC changes slowly with temperature starting from around $400 \mathrm{~K}$ in agreement with predictions for two-dimensional transition metal dichalcogenides [36,37]. Different from $\mathrm{MoS}_{2}$, we do not observe any saturation of the TEC for black $P$ at high temperatures. While black P expands at most $0.02 \AA$ along the zigzag direction as $T$ approaches $1000 \mathrm{~K}$, it expands $0.04 \AA$ along the armchair direction. As is clear from Fig. 1, a uniaxal expansion along the armchair direction may result in a structural phase transition from black $\mathrm{P}$ to blue $\mathrm{P}$ because of the similar hexagonal arrangement of $\mathrm{P}$ atoms [44]. Here, it is worth mentioning that the validity of the results at high temperature may be questionable because it depends on the anharmonic nature of the crystal. The quasiharmonic approximation (QHA) neglects anharmonicity resulting from the intrinsic phonon-phonon interaction. However, previous studies have shown that QHA gives quite accurate results for the in-plane lattice TEC of graphite [35] and lattice TEC of diamond [37] crystals up to $1000 \mathrm{~K}$.

In Fig. 5, the variation of the cohesive energy as a function of strain at $0 \mathrm{~K}$ (a) and the variation of the Helmholtz free energy $[F(T)]$ as a function of temperature (b) are presented. In Fig. 5(b), we also show $\Delta F(T)$ which is defined as $\Delta F(T)=$ $F_{\text {blue } P}(T)-F_{\text {black } P}(T)$. Here, $F_{\text {blue } P}(T)$ and $F_{\text {black } P}(T)$ are the Helmholtz free energy of black P and blue P, respectively. 

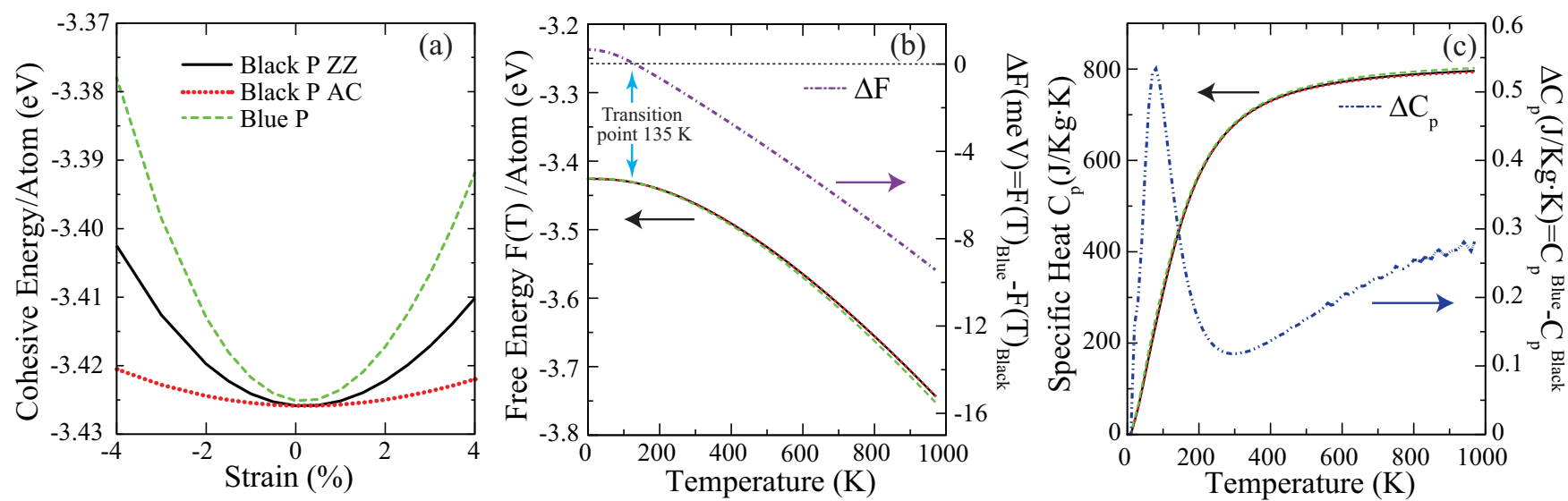

FIG. 5. (Color online) (a) Cohesive energy, including zero-point energy, as a function of applied strain at $T=0 \mathrm{~K}$ and (b) Helmholtz free energy $[F(T)]$ as a function of temperature. Here, $\mathrm{ZZ}$ and $\mathrm{AC}$ are used for the zigzag and armchair directions, respectively. $\Delta F(T)$ is the difference between $F(T)$ of black P and blue P. The blue arrow (b) denotes the transition point, after which blue P becomes thermodynamically more stable over black P. The specific heat at constant pressure $\left(C_{p}\right)$ and the difference $\left(\Delta C_{p}\right)$ between $C_{p}$ of black $\mathrm{P}$ and blue $\mathrm{P}$ are shown in (c).

As the zero-point energy continuously decreases with strain from minus to plus, the variation of the zero-point energy results in an asymmetric behavior in cohesive energy with strain. In addition, the curvature of this total energy gives the in-plane stiffness of the material as a measure of the response to mechanical deformation. It is clear that blue $\mathrm{P}$ is a stiffer material as compared to black P. Moreover, the deformation along the zigzag direction of black $\mathrm{P}$ is harder than that along the armchair direction at $0 \mathrm{~K}$, and this is consistent with the finite-temperature behavior as we conclude from the previous section in connection with Fig. 4(a).

The $F(T)$ decreases as temperature increases due to the entropy term [the last term in Eq. (1)]. Inclusion of the zero-point energy gives rise to a slightly higher ground state energy for blue P over black P. However, as temperature increases, a crossing of the free-energy curves around $135 \mathrm{~K}$ occurs, which makes the blue $\mathrm{P}$ energetically more favorable at high temperatures; see Fig. 5(b). The free-energy difference between the two phases is of the order of $1 \mathrm{meV} /$ atom at room temperature, meaning that the two phases can coexist. In addition, it is possible to observe a phase transition driven by temperature from black $\mathrm{P}$ to blue $\mathrm{P}$ or vice versa at $T=135 \mathrm{~K}$.

In Fig. 5(c), we present constant-pressure heat capacity, $C_{p}$, results for both structures, which is generally a few percent different from constant-volume heat capacity in similar structures [35]. The $C_{p}$ difference $\left(\Delta C_{p}\right)$ between two phases is significantly small for all temperature ranges as represented with the blue dash-dotted line in Fig. 5(c), which states essentially very similar Debye temperature for these two different phases. At high temperatures, $C_{p}$ approaches its classical value of $3 k_{B} /$ atom. When $T=300 \mathrm{~K}, C_{p}$ already reaches about $80 \%$ of its classical value, meaning that most of the phonon modes are activated at this temperature.

In summary, we systematically investigate the lattice thermal properties of black and blue P. Similar to its electronic properties, black $\mathrm{P}$ has direction-dependent mechanical and thermal properties. Our results show that the frequency shift observed in particular modes can be utilized to determine strain distribution in phosphorene. The calculated thermal expansion coefficients demonstrate that a much larger expansion along the armchair direction with temperature is observed for black $P$. While black $\mathrm{P}$ is thermodynamically more stable than blue $\mathrm{P}$, the latter becomes more stable when $T>135 \mathrm{~K}$. Due to their structural similarities, it is possible to observe a structural phase transition from black $\mathrm{P}$ to blue $\mathrm{P}$ by increasing temperature beyond $135 \mathrm{~K}$, and therefore the coexistence of these two phases is possible.

This work was supported by the Flemish Science Foundation (FWO-V1) and the Methusalem foundation of the Flemish government. Computational resources were provided by TUBITAK ULAKBIM, High Performance and Grid Computing Center (TR-Grid e-Infrastructure), and HPC infrastructure of the University of Antwerp (CalcUA), a division of the Flemish Supercomputer Center (VSC), which is funded by the Hercules foundation. C.S. acknowledges the support from Anadolu University (BAP-1407F335), and Turkish Academy of Sciences (TUBA-GEBIP).
[1] H. Liu, A. T. Neal, Z. Zhu, Z. Luo, X. Xu, D. Tománek, and P. D. Ye, ACS Nano 8, 4033 (2014).

[2] L. Li, Y. Yu, G. J. Ye, Q. Ge, X. Ou, H. Wu, D. Feng, X. H. Chen, and Y. Zhang, Nat. Nanotechnol. 9, 372 (2014).
[3] Z. Zhu and D. Tománek, Phys. Rev. Lett. 112, 176802 (2014).

[4] J. Guan, Z. Zhu, and D. Tománek, Phys. Rev. Lett. 113, 046804 (2014).

[5] M. Wu, H. Fu, L. Zhou, K. Yao, and X. C. Zeng, Nano Letters 15, 3557 (2015). 
[6] D. Çakır, H. Sahin, and F. M. Peeters, Phys. Rev. B 90, 205421 (2014).

[7] X. Wang, A. M. Jones, K. L. Seyler, V. Tran, Y. Jia, H. Zhao, H. Wang, L. Yang, X. Xu, and F. Xia, Nat. Nanotechnol. 10, 517 (2015).

[8] Y. Maruyama, S. Suzuki, K. Kobayashi, and S. Tanuma, Physica B +C 105, 99 (1981).

[9] S. Narita, Y. Akahama, Y. Tsukiyama, K. Muro, S. Mori, S. Endo, M. Taniguchi, M. Seki, S. Suga, A. Mikuni, and H. Kanzaki, Physica B+C 117, 422 (1983).

[10] J. Qiao, X. Kong, Z.-X. Hu, F. Yang, and W. Ji, Nat. Commun. 5, 4475 (2014).

[11] J. Dai and X. C. Zeng, J. Phys. Chem. Lett. 5, 1289 (2014).

[12] A. S. Rodin, A. Carvalho, and A. H. Castro Neto, Phys. Rev. Lett. 112, 176801 (2014)

[13] R. Fei and L. Yang, Nano Lett. 14, 2884 (2014).

[14] S. Appalakondaiah, G. Vaitheeswaran, S. Lebegue, N. E. Christensen, and A. Svane, Phys. Rev. B 86, 035105 (2012).

[15] R. Fei and L. Yang, Appl. Phys. Lett. 105, 083120 (2014).

[16] M. Elahi, K. Khaliji, S. M. Tabatabaei, M. Pourfath, and R. Asgari, Phys. Rev. B 91, 115412 (2015).

[17] Y. Feng, J. Zhou, Y. Duand, F. Miao, C.-G. Duan, B. Wang, and X. Wan, J. Phys.: Condens. Matter 27, 185302 (2015).

[18] V. Tran, R. Soklaski, Y. Liang, and L. Yang, Phys. Rev. B 89, 235319 (2014).

[19] A. S. Rodin, A. Carvalho, and A. H. Castro Neto, Phys. Rev. B 90, 075429 (2014).

[20] A. Chaves, T. Low, P. Avouris, D. Çakır, and F. M. Peeters, Phys. Rev. B 91, 155311 (2015).

[21] D. Çakır, C. Sevik, and F. M. Peeters, arXiv:1506.04707 [condmat.mes-hall].

[22] L. Seixas, A. S. Rodin, A. Carvalho, and A. H. Castro Neto, Phys. Rev. B 91, 115437 (2015).
[23] M. Mehboudi, K. Utt, H. Terrones, E. O. Harriss, A. A. Pacheco San Juan, and S. Barraza-Lopez, Proc. Natl. Acad. Sci. USA 112, 5888 (2015).

[24] Z.-Y. Ong, Y. Cai, G. Zhang, and Y.-W. Zhang, J. Phys. Chem. C 118, 25272 (2014).

[25] A. Jain and A. J. H. McGaughey, Sci. Rep. 5, 8501 (2015).

[26] G. Kresse and J. Hafner, Phys. Rev. B 47, 558 (1993).

[27] G. Kresse and J. Hafner, Phys. Rev. B 49, 14251 (1994).

[28] G. Kresse and J. Furthmuller, Comput. Mater. Sci. 6, 15 (1996).

[29] G. Kresse and J. Furthmuller, Phys. Rev. B 54, 11169 (1996).

[30] P. E. Blöchl, Phys. Rev. B 50, 17953 (1994).

[31] G. Kresse and D. Joubert, Phys. Rev. B 59, 1758 (1999).

[32] J. P. Perdew, K. Burke, and M. Ernzerhof, Phys. Rev. Lett. 77, 3865 (1996).

[33] J. P. Perdew, K. Burke, and M. Ernzerhof, Phys. Rev. Lett. 78 , 1396 (1997).

[34] A. Togo, F. Oba, and I. Tanaka, Phys. Rev. B 78, 134106 (2008).

[35] N. Mounet and N. Marzari, Phys. Rev. B 71, 205214 (2005).

[36] D. Çakır, F. M. Peeters, and C. Sevik, Appl. Phys. Lett. 104, 203110 (2014).

[37] C. Sevik, Phys. Rev. B 89, 035422 (2014).

[38] A. Togo, L. Chaput, I. Tanaka, and G. Hug, Phys. Rev. B 81, 174301 (2010).

[39] P. Pavone, K. Karch, O. Schütt, D. Strauch, W. Windl, P. Giannozzi, and S. Baroni, Phys. Rev. B 48, 3156 (1993).

[40] B. Sa, Y.-L. Li, J. Qi, R. Ahuja, and Z. Sun, J. Phys. Chem. C 118, 26560 (2014)

[41] C. Kittel, Introduction to Solid State Physics (John Wiley \& Sons, Inc., New York, 2004).

[42] M. Neek-Amal and F. M. Peeters, Phys. Rev. B 83, 235437 (2011).

[43] J. Brivio, D. T. L. Alexander, and A. Kis, Nano Lett. 11, 5148 (2011).

[44] J.-W. Jiang and H. S. Park, Nat. Commun. 5, 4727 (2014). 